\title{
TV/Series
}

$17 \mid 2020$

Séries : les sens de l'Histoire

\section{Les séries télévisées médicales étatsuniennes : évolutions, permanences et enjeux de l'« asymétrie réaliste »}

\section{Elisabeth Fauquert}

\section{(2) OpenEdition \\ Journals}

Édition électronique

URL : http://journals.openedition.org/tvseries/4296

DOI : $10.4000 /$ tvseries.4296

ISSN : 2266-0909

Éditeur

GRIC - Groupe de recherche Identités et Cultures

\section{Référence électronique}

Elisabeth Fauquert, «Les séries télévisées médicales étatsuniennes : évolutions, permanences et enjeux de l'« asymétrie réaliste »», TV/Series [En ligne], 17 | 2020, mis en ligne le 24 juin 2020, consulté le 10 décembre 2020. URL : http://journals.openedition.org/tvseries/4296 ; DOI : https://doi.org/ $10.4000 /$ tvseries.4296

Ce document a été généré automatiquement le 10 décembre 2020.

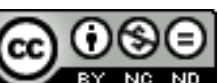

TV/Series est mis à disposition selon les termes de la licence Creative Commons Attribution - Pas d'Utilisation Commerciale - Pas de Modification 4.0 International 


\title{
Les séries télévisées médicales étatsuniennes : évolutions, permanences et enjeux de l'« asymétrie réaliste »
}

\author{
Elisabeth Fauquert
}

\section{Introduction}

1 Les séries médicales sont nées avec la télévision, ont évolué avec elle et avec la société qu'elles entendent représenter. Le franc succès de productions étatsuniennes telles que Medic (NBC, 1954-1956), Dr Kildare (NBC, 1960-1966) et Ben Casey (ABC, 1961-1966) est noté dès le début, comme en atteste l'extrait suivant tiré d'un ouvrage de Marshall MacLuhan paru en 1964 :

En créant une passion pour une exploration en profondeur de tous les aspects de l'expérience, l'image télévisuelle crée une obsession pour le bien-être du corps. L'émergence soudaine de la série télévisée médicale hospitalière comme rivale du western est parfaitement naturelle ${ }^{1}$.

2 La série médicale aurait donc "naturellement", rapidement acquis ses lettres de noblesse dans le paysage sériel. Elle figure jusqu'à ce jour parmi les genres les plus populaires de la télévision (plusieurs millions de téléspectateurs pour chaque épisode de chaque série), succès ininterrompu qui doit sans doute à la plasticité du genre qui emprunte aux influences classiques comme la comédie (avec le hospital sitcom Scrubs, NBC/ABC 2001-2010), le soap opera (avec le hospital soap Grey's Anatomy, ABC, 2005 -) ou encore la série policière (avec le hospital whodunit Dr House, Fox, 2004-2012).

Les séries médicales faisant partie du canon sériel au même titre que les séries policières, il n'est guère étonnant que ce genre ait suscité et continue de susciter l'intérêt des chercheurs. Nombre de travaux sur le sujet utilisent le cadre théorique des fictions à substrat professionnel (FASP), telles que les a identifiées Michel Petit ${ }^{2}$. Les 
chercheurs ayant choisi ce cadre ont étudié la collaboration de spécialistes (médecins, infirmières) à l'élaboration des scénarios, la valeur pédagogique de ce genre, l'authenticité des contenus ou encore la portée du discours médical spécialisé3. D'autres chercheurs ont étudié finement les ressorts scénaristiques, le réalisme et la vraisemblance de séries comme Urgences (NBC, 1994-2009) ou Dr House ${ }^{4}$.

4 Si l'étude du réalisme et de la vraisemblance du genre occupe une place de choix dans les recherches actuelles, à l'inverse, l'évolution historique, diachronique des choix de focalisation dans les séries médicales (choix de l'unité de lieu, sociologie des personnages principaux, discours relayés, etc.) n'a presque pas été étudiée. L'absence de recherche sur ce point est sans doute à relier à la remarquable continuité des choix de focalisation depuis l'apparition du genre sur le petit écran : depuis les années 1950, la focalisation dominante est celle d'une intrigue suivant une équipe d'internes et/ou d'urgentistes dans un hôpital urbain. La continuité de ces choix de focalisation est-elle problématique pour un genre aussi populaire? Quels sont les enjeux épistémiques de ces choix de focalisation, $a$ fortiori dans le contexte de réception étatsunien, où l'accès limité aux soins médicaux demeure un enjeu de premier plan, souvent de vie ou de mort?

5 Cet article explore ces interrogations liminaires et a vocation à compléter les recherches actuelles en étudiant le paradoxe de «l'asymétrie réaliste » dans les séries médicales étasuniennes et ses enjeux. L' " asymétrie réaliste » est ici entendue comme la tension entre deux pôles de l'économie dramatique des séries hospitalières étatsuniennes. Le premier est l'hypervisibilité de la science et de l'expertise médicale, inscrite dans un " présentisme " propre au genre (le présentisme étant ici entendu au sens d'Hartog, à savoir la représentation d'un zeitgeist hermétique, sorte de gros plan très détaillé, d'éternel présent isolé des influences passées et coupé des projections futures ${ }^{5}$.) L'autre pôle est l'invisibilité (ou plutôt l'invisibilisation) des inégalités d'accès aux soins médicaux, question souvent reléguée au hors champ. Plus concrètement, et pour reprendre les termes d'André Bazin, l'article pose que l'asymétrie réaliste des séries médicales étatsuniennes est à la fois un «cadre » et un «cache» de la réalité sociale dans l'arène de la santé ${ }^{\text {. }}$

6 Le protocole de recherche utilisé pour cet article est inductif. Il s'appuie sur un corpus de séries médicales produites aux États-Unis sur la période 1954-2018 comme Dr Kildare (NBC, 1960-1966), Ben Casey (ABC, 1961-1966), Urgences, Grey's Anatomy, Dr House et Scrubs, et fait aussi le pari d'inclure d'autres séries qui traitent directement ou indirectement de la thématique de la santé comme Friends (NBC, 1994-2004) et Breaking Bad (AMC, 2008-2013). L'argumentaire analyse les choix de focalisation dans les séries mentionnées ci-avant (choix du cadre de l'action, choix des protagonistes principaux, choix des discours que ces derniers tiennent à travers le temps); ces choix de focalisation sont en outre contextualisés, complétés et situés grâce à des éclairages pluridisciplinaires (histoire, études civilisationnelles, sociologie des médias, etc.).

7 Ce travail est divisé en deux sections. La première étudie la série médicale en tant que cadre : y est analysée la genèse du canon sériel médical et ses évolutions. La deuxième section analyse les enjeux épistémiques de la focalisation dans les séries médicales, pour montrer qu'elles fonctionnent aussi comme un cache, deuxième facette de l'asymétrie réaliste présentée en introduction. 


\section{I) La série médicale, un cadre : genèse et variations d'un canon}

8 Chaque série est caractérisée par ce que Sarah Sepulchre nomme "le retour du même ${ }^{7}$. » À l'échelle micro, ce retour du même renvoie à un univers, à des intrigues et à des personnages que le téléspectateur côtoie dans le temps long d'une série. À l'échelle macro, le retour du même peut s'entendre comme la familiarité du téléspectateur avec un genre télévisuel, résultant d'une exposition répétée à des contenus codifiés produits à la télévision (television literacy). Dans le cas précis des séries médicales, ce retour du même (à l'intérieur d'une même série et dans le cadre plus large de la television literacy) a donné naissance à un genre autonome, terme entendu ici au sens de Schaeffer, à savoir une "catégorie de classification rétrospective ${ }^{8}$." Nous proposons que de simple "genre », la série médicale s'est transformée en un canon, tantôt conforté, tantôt subverti. Ce canon repose sur trois choix de focales que nous étudions dans les sections qui suivent : une intrigue dont les médecins sont la focale et les héros (1), une intrigue prenant pour focale l'univers fictif de l'hôpital en milieu urbain (2) et enfin une focalisation sur le langage médical spécialisé (3).

\section{a) Le médecin comme focale : naissance d'un héros de la culture populaire}

9 Le médecin est aujourd'hui une focale «naturelle » de la culture populaire de fiction, et des séries médicales en particulier. Il convient cependant de rappeler que l'entrée du médecin au panthéon des héros de fiction aurait été impensable il y a 150 ans, car l'ascension sociale des médecins est un phénomène récent. En effet, tout au long du xix siècle, la médecine était une profession méprisée. Les chirurgiens jouissaient d'un prestige scientifique et social quasi-nul. Aux États-Unis, le pouvoir social, politique et scientifique des médecins ne se consolide qu'à partir des années 1910 avec l'avènement de la chimie de synthèse et de la médecine de laboratoire, les développements de l'anesthésie, de la bactériologie et de la chirurgie antiseptique, qui toutes accélèrent la professionnalisation de la médecine, et fondent la légitimité des praticiens, leur autorité et leur aura dans la société étatsunienne.

Cette ascension sociale progressive ne suffit toutefois pas à hisser la profession au rang d'icône : les médecins pâtissent d'une faible notoriété publique jusqu'aux années 1930. Au cours de cette décennie, le plus puissant lobby médical d'alors, l'American Medical Association (ci-après AMA), fait pression sur les scénaristes d'Hollywood et leur intime de redorer une fois pour toutes le blason de la profession. C'est chose faite à l'aide de films à succès comme Men in White (Metro-Goldwyn-Mayer, 1934), A Man to Remember (RKO Radio Pictures, 1938) ou encore Dark Victory (Warner Bros, 1939). C'est dans ce contexte qu'apparait le premier médecin fictionnel célèbre, le Dr Kildare, héros de plusieurs films à succès dans les années 1940. Les séries médicales produites au début des années 1950 s'inscrivent dans ce sillage. Ces productions dépeignent les médecins (et les chirurgiens en particulier) en chevaliers de la croisade moderne de la science contre la mort, ce qui contribue à l'ascension sociale des vrais médecins. Des programmes comme The Doctor (NBC, 1952-1953), City Hospital (CBS, 1952-1953) et surtout Medic, première série médicale diffusée en prime time aux États-Unis, rencontrent un franc succès. Forte de cette réussite, la chaîne NBC produit une 
nouvelle série médicale, $\mathrm{Dr}$ Kildare (NBC, 1960-1966); ABC produit une série concurrente, Ben Casey (ABC, 1961-1966). Toutes deux figurent parmi les séries les plus populaires des années 1960. Dr Kildare et Ben Casey, toutes deux créées par le scénariste James Moser, sont les prototypes du canon sériel médical : elles établissent des focales qui continuent de régir ce genre de nos jours : Dr Kildare suit le parcours professionnel de James Kildare, jeune interne en médecine au Blair General Hospital, et véhicule une représentation idéalisée du médecin, figure virile (toujours masculine à l'époque), séduisante, infaillible et pleine de compassion.

11 La représentation des médecins dans les séries comme Dr Kildare et Ben Casey et la circulation de cette image est très soigneusement contrôlée. Ce contrôle tient tout d'abord à la structure du réseau télévisé des années 1960, âge d'or du réseau hertzien et de la domination de trois réseaux de télévision terrestre (the Big Three) : $\mathrm{ABC}, \mathrm{NBC}$ et CBS. Si le réseau offre un choix limité, l'exposition des téléspectateurs, elle, est considérable: en 1960, $87 \%$ des foyers étatsuniens sont équipés d'un poste de télévision, soit un public potentiel de 46 millions de téléspectateurs ${ }^{9}$. Ce contrôle tient également à l'AMA. Le lobby, qui détenait déjà un droit de regard sur les scénarios au nom du réalisme médical, est allé plus loin en imposant un catéchisme professionnel aux personnages de fiction, pour asseoir le statut et la réputation des médecins dans la société. Joseph Turow explique ainsi que les docteurs de fiction n'étaient pas autorisés à s'asseoir sur les lits de leurs patientes, pas plus qu'ils n'étaient autorisés à conduire des voitures de luxe ou à plaisanter sur le sort des malades ${ }^{10}$.

Enfin, ce contrôle tient au contexte de propagande scientifique de Guerre froide, qui contraint les États-Unis à se (re)présenter en patrie du progrès scientifique éclairé. Dans les années 1960, les médecins du petit écran ne font aucune erreur médicale et ne sont la proie d'aucun doute. Les séries de l'époque ne s'intéressent pas tant à la vie personnelle des soignants qu'au désintéressement héroïque avec lequel les médecins remplissent leur mission. La plupart des épisodes montrent d'ailleurs la fatigue harassante qui accable les internes et sert de piédestal à leur abnégation. Ce métadiscours n'a pas disparu, et constitue encore aujourd'hui l'une des recettes diégétiques de séries médicales comme Urgences ou Grey's Anatomy. Pour ne citer qu'un exemple de ces continuités, voici le texte que Meredith Grey, principale protagoniste de la série Grey's Anatomy, prononce en voix off en conclusion d'un épisode au titre explicite, «The Self-Destruct Button » (saison 1, épisode 7):

Ok. J'aimerais bien les voir après quelques mois d'internat, ceux qui me disent qu'on dormira quand on sera mort. Alors oui, ce n'est pas simplement notre métier qui nous tient éveillés toute la nuit. Je veux dire, la vie est déjà tellement dure, pourquoi s'ajouter des difficultés? D'où nous vient cette nécessité d'appuyer sur le bouton « auto-destruction »? Peut-être qu'on aime la douleur. Peut-être qu'on est fait comme ça. Parce que sans elle, et bien, peut-être qu'on ne se sentirait pas vivants. Qu'est-ce que ça veut dire ? Pourquoi est-ce que je continue à me taper sur la tête avec un marteau? Parce que ça fait tellement de bien quand j'arrête ${ }^{11}$.

Dans les séries des années 1960, l'image et la représentation du médecin à la télévision est donc parfaitement maîtrisée: elle est unidimensionnelle, héroïque, presqu'inhumaine de perfection, de rigueur et d'éthique. Bien que cette représentation perdure souvent dans les séries médicales contemporaines (comme le montre l'extrait de Grey's Anatomy précité), la représentation des médecins à l'écran s'est complexifiée ces dernières décennies. 


\section{b) Les médecins du petit écran : contestations, réécritures} comme les virologues et les épidémiologistes, brisant le monopole que les médecins détenaient sur la profession ${ }^{12}$. Le scandale de la thalidomide en Europe et au Canada ébranle le prestige de la profession à l'international (même si très peu de cas de malformations sont recensés aux États-Unis). Hors du champ strictement scientifique, les forces de la société civile, de la contre-culture et des mouvements sociaux (mouvement pour les droits civiques, mouvements féministes, mouvements pour les droits des patients) redessinent peu à peu le périmètre de l'autorité médicale ${ }^{13}$ et le réduisent. fictionnels. Les médecins des séries actuels sont moins monolithiques que leurs archétypes du passé. Les praticiens sont humanisés : ils sont confrontés aux mêmes difficultés que les téléspectateurs, comme la mort d'un parent, l'addiction à la drogue, les maladies, les échecs sentimentaux, etc.

Par exemple, dans la première saison de Grey's Anatomy (saison 1, épisode 9) les internes doivent faire face à une épidémie de syphilis qui se déclare à cause de l'interne Alex Karev. Toujours dans cette première saison, l'interne Cristina Yang tombe enceinte mais ne souhaite pas garder l'enfant, et parle de son choix de recourir à l'IVG. Enfin, bien que la plupart des internes de Grey's Anatomy aient grandi dans des familles aisées, d'autres sont issus de milieux modestes, voire de familles dysfonctionnelles. On apprend ainsi que l'interne Izzie Stevens a payé ses frais d'inscription en faculté de médecine en posant pour des photos de lingerie et que l'interne Alex Karev a été placé en famille d'accueil à dix-sept reprises parce que sa mère était schizophrène et son père alcoolique. Cette humanisation des médecins se retrouve dans la série Urgences, comme le note Sabine Chalvon-Demersay. Le pilote de la série met en lumière les faiblesses des médecins, chose impensable dans les séries des années 1950 et 1960. La série s'ouvre en effet sur une scène montrant le docteur Greene assoupi dans la salle de repos des internes. Une infirmière le réveille pour lui annoncer un patient. Ce patient n'est autre que le docteur Doug Ross (incarné par George Clooney), qui s'est présenté ivre mort à l'accueil des urgences hospitalières. Cette scène illustre l'évolution de la représentation du médecin en ce qu'elle abolit la relation hiérarchique médecin-patient pour donner à voir ce que Chalvon-Demersay nomme un "entrelacement des conditions ${ }^{14}$." Cet «entrelacement » est d'autant plus fort que le docteur Ross est un des principaux protagonistes de la série Urgences et donc l'une de ses principales focales dramatiques.

La déconstruction de la figure idéalisée du médecin est poussée un cran plus loin dans la série Dr House, qui subvertit totalement le catéchisme professionnel hérité des années 1960. En effet, comme le note Pamela Tytell, « Gregory House casse le stéréotype étroit et figé du médecin idéal, le héros fort et courageux ${ }^{15}$. » Le signe le plus notable de l'anticonformisme de House est sans doute son refus de porter la blouse blanche, marqueur iconique, métonymique de son autorité scientifique et de son prestige social. À ce choix s'ajoute des traits de personnalité étonnants en regard des anciens codes du genre: House est un anti-héro brusque, machiavélique, dépendant à la Vicodin, un soignant qui ne s'encombre pas du décorum (il mange dans la morgue, ce qui est interdit) et prescrit des remèdes hétérodoxes, comme lorsqu'il conseille à un homme 
déguisé en Père Noël de fumer plusieurs cigarettes par jour pour guérir ses coliques intestinales (saison 1, épisode 5).

\section{c) Entrées dans le cadre : médecins et diversités}

18 La réécriture réaliste des médecins de fiction passe aussi par l'entrée dans le cadre de profils jusque-là relégués au hors champ (femmes médecins, médecins racisés), bien que la focale sur les internes et urgentistes demeure, elle, inchangée.

19 Cette évolution des codes du genre apparaît par exemple dans Grey's Anatomy, série créée par la productrice africaine-américaine Shonda Rimes. Par le passé, les stéréotypes sur les femmes étaient nombreux dans les séries médicales, de Dr Kildare à Urgences. Comme le souligne Jason Jacobs,

jusqu'à récemment, les femmes étaient présentées négativement dans les séries médicales : lorsqu'elles ne sont pas l'objet de désir des médecins, elles sont privées de leurs attributs féminins [...]. On les présente comme mues par leurs seuls intérêts, comme des personnages rigides et procéduriers, à qui l'on confisque toute empathie et toute liberté ${ }^{16}$.

20 À l'inverse, la principale protagoniste de Grey's Anatomy, l'interne Meredith Grey, est une femme et la fille d'une chirurgienne célèbre. La série va même plus loin dans le renversement des codes du passé, puisque les internes comme Meredith, Izzie et Cristina objectivent les médecins hommes en les affublant de sobriquets comme "McDreamy » (« docteur Mamour » en français, surnom du docteur Derek Shepherd) ou « McSteamy " (« docteur Glamour » en français, surnom du docteur Mark Sloane). Ces surnoms sont un clin d'œil aux codes du genre, qui a traditionnellement érigé les acteurs principaux en sex-symbols, qu'il s'agisse de Richard Chamberlain dans $\mathrm{Dr}$ Kildare, de George Clooney dans Urgences ou encore de Patrick Dempsey dans Grey's Anatomy.

21 Enfin, dans Grey's Anatomy, le chef de service, le Dr Richard Weber et la chef des internes Miranda Bailey sont noirs. D'autres protagonistes centraux sont issus des minorités comme Calliope Torres (hispano-étatsunienne) ou Cristina Yang (coréenneétatsunienne). On voit aussi davantage de médecins LGBT depuis les années 1990: Urgences fut la première série télévisée en prime time à inclure une médecin lesbienne ; une partie de l'intrigue des saisons 5 à 9 de Grey's Anatomy est consacrée à l'histoire d'amour entre deux médecin, Arizona Robbins et Calliope Torres.

En somme, la représentation contemporaine des médecins dans les séries télévisées médicales remplit une fonction symbolique, ce que François Jost nomme « universalité anthropologique. » Pour Jost, le succès des séries contemporaines tient au fait qu'elles « racontent l'histoire de personnages du mode mimétique bas, c'est-à-dire de personnages qui nous ressemblent ${ }^{17}$ ", là où les feuilletons d'hier donnaient à voir des héros invincibles, présentés comme supérieurs au public (mode mimétique haut). En dépit de l'extranéité professionnelle et linguistique des mondes fictifs des séries médicales, les failles des protagonistes les rattachent incontestablement à un destin et à une condition humaine partagés, qui les rapprochent des téléspectateurs ${ }^{18}$. Ce rapprochement dans l'humanité n'abolit cependant pas toutes les distances, en particulier le fait que les protagonistes évoluent dans un univers dont ils détiennent les codes, celui de l'hôpital urbain, deuxième focale du canon des séries médicales. 


\section{II) L'hôpital urbain : une focale dramatique} ancré dans la célébration des « miracles » de la médecine moderne et des performances de l'institution qui en compose le cœur : l'hôpital étatsunien. Le réseau hospitalier se développe de manière considérable après l'adoption de la loi Hill-Burton (1946) qui attribue des subventions et des prêts concurrentiels aux États pour promouvoir la construction d'hôpitaux. Cette loi permet l'expansion du réseau hospitalier, mais n'améliore que de manière limitée l'accès aux soins pour les plus pauvres, notamment dans les zones rurales. En outre, les dispositions ségrégationnistes de cette loi ont entériné plus avant le développement séparé des institutions médicales. Malgré ces inégalités, l'hôpital sert de prisme au rayonnement scientifique et à l'avant-garde médicale, dans les temps mouvementés de la Guerre froide, de la conquête spatiale et de la course aux armements.

À cette époque, l'hôpital n'est pas seulement le locus du rayonnement scientifique. Il est également présenté comme le microcosme et le conservatoire de valeurs morales et normatives comme le travail, le mérite et la rédemption, valeurs dont les médecins sont la métonymie. On peut, dans la même optique, proposer que le travail en équipe dans les séries médicales des années 1960 est le substitut allégorique de la famille nucléaire traditionnelle. En effet, les séries de cette époque mettent souvent en scène les tensions entre une jeune garde idéaliste et ses supérieurs, même si les différends sont toujours apaisés à l'aune de la relation bienveillante entre l'interne et la figure paternelle du chef de service, qu'incarne par exemple le docteur Leonard Gillepsie dans Dr Kildare. La dynamique est légèrement différente dans Ben Casey, série plus sombre qui montre un conflit inter-générationnel annonciateur de la contre-culture à travers des démêlés entre le héros rebelle et ses supérieurs plutôt bornés appartenant à l'establishment médical.

\section{b) Crise de l'hôpital : terreau de réécritures}

La dimension morale et normative de l'unité de lieu hôpital a bien entendu évolué au gré des évolutions de la société et du système de santé dans son ensemble.

La sitcom culte $M^{*} A^{*} S^{*} H^{* 20}$ (Fox/CBS 1972-1983) marque une première rupture décisive dans les représentations de l'hôpital étatsunien. Dans cette série comique et dramatique prenant la guerre de Corée (1950-1953) pour théâtre et faisant écho au bourbier vietnamien, l'hôpital militaire mobile est présenté comme un sanctuaire absurde où les médecins ne disposent d'aucun contrôle sur leur environnement de travail et se détournent facilement de leurs missions de soignants. 
28 En parallèle et à la même période (années 1970), les hôpitaux sont confrontés à une crise durable de financement. En effet, de nombreux établissements refusent de prendre en charge les patients couverts par Medicaid (le programme d'assurance maladie pour les plus pauvres) et la construction de nouveaux hôpitaux est soumise à l'obtention d'un certificat de nécessité (Certificate of Need), qui confère un monopole de fait aux hôpitaux déjà en place dans une région donnée. De nombreuses unités spéciales, comme les urgences médicales, les centres pour prématurés ou les unités de traitement des grands brûlés, ferment leurs portes pour réduire les coûts.

Autre signe manifeste d'inégalités, l'exclusion des patients pauvres se banalise (patient dumping) ${ }^{21}$. En effet, de nombreux hôpitaux demandent un paiement ou un acompte avant toute consultation ou toute intervention, refusent d'admettre les patients qui ne sont pas suivis personnellement par un médecin de l'hôpital où il/elle se présente. En conséquence, les urgences d'un hôpital refusent parfois de soigner un malade ou de prendre en charge une femme en travail, car il/elle ne peut payer ou n'a pas d'assurance maladie qui rembourse les actes médicaux nécessités. Il en va de même pour les patients infectés au VIH, en état d'ébriété ou en overdose, qui sont aussi généralement exclus.

Ces inégalités ne passent pas sous silence et conduisent à l'adoption d'une loi fédérale anti-dumping, la loi EMTALA, adoptée en 1986. Cette loi (toujours en vigueur) oblige les services des urgences hospitalières et les ambulances à prendre en charge les personnes nécessitant un traitement d'urgence, et ce indépendamment de leur capacité à payer, de leur nationalité ou de leur statut légal. Les hôpitaux et les médecins contrevenants s'exposent à des pénalités financières de 50000 dollars par cas ignoré.

\section{c) Les urgences hospitalières : sanctuaire égalitaire et caution morale de la société étatsunienne?}

31 C'est dans ce contexte qu'il faut désormais envisager l'unité de lieu hôpital dans les séries médicales contemporaines. D'abord, il n'est pas anodin que les hôpitaux fictionnels présentés dans les séries soient toujours des hôpitaux urbains desservant les populations des grandes métropoles, où le « danger » est pour ainsi dire permanent : le Blair General Hospital dans Dr Kildare, l'hôpital universitaire de Cook County dans Urgences, le Sacred Heart Hospital dans Scrubs, l'hôpital Seattle Grace dans Grey's Anatomy ou encore le Princeton-Plainsboro Teaching Hospital dans Dr House. Ensuite, il n'est pas fortuit que les séries les plus récentes, comme Urgences ou Grey's Anatomy, aient pour cadre les urgences hospitalières. Les urgences sont une sorte de passerelle depuis le monde extérieur vers l'univers hospitalier, espace de rationalité et de stabilité qui sert de contrepoint à la brutalité des accidents, des maladies et de la mort, un lieu où la violence physique et symbolique (de la vie urbaine notamment) peut être maîtrisée, apprivoisée et apaisée.

Le contraste (et la dialectique) entre un extérieur/hors-champ hostile et un intérieur/ cadre technique et maitrisé sert de nœud dramatique à tous les épisodes du genre. Ce contraste apparaît de manière encore plus frappante quand la violence fait irruption dans ce milieu protégé et sanctuaire. C'est notamment le cas des séries Urgences et Grey's Anatomy, qui ont chacune un épisode où un tueur armé pénètre dans l'enceinte de l'hôpital et tire à vue. Par exemple, dans le bien nommé épisode « Sanctuary » de Grey's Anatomy (saison 6, épisode 23), Gary Clark, époux d'une patiente décédée à l'hôpital 
Seattle Grace, entre armé dans l'hôpital prêt à venger sa mort en abattant les médecins Derek Shepherd, Richard Webber et Lexie Grey.

Enfin, les séries présentent presque toujours l'hôpital comme une sorte de sanctuaire égalitaire. D'abord parce que les urgences hospitalières, qui ne peuvent plus refuser de patients, se sont de facto transformées en filet de sécurité pour l'ensemble de la population, assurée ou non, riche ou non, citoyenne ou non. Ensuite, le potentiel égalitaire de l'hôpital sanctuaire tient au fait que l'accident et la maladie peuvent frapper tout un chacun à tout instant, indépendamment de son statut social ou de sa couleur de peau. Les médecins sont les garants démocratiques de l'universalité de la vulnérabilité humaine, et la série médicale s'en fait le relais. Cette dimension démocratique est enfin renforcée par le médium qui les transmet, la télévision étant par nature un médium démocratique, "dont l'accès ne repose sur aucune discrimination par la fortune ou la culture ", comme le souligne Patrick Lecomte ${ }^{22}$.

En un mot, la focalisation sur l'hôpital-sanctuaire tiendrait lieu de caution morale et démocratique à la société étatsunienne, dans une arène de la santé par ailleurs profondément inégalitaire. Nous abordons à présent la dernière dimension du canon du genre, la centralité du discours médical spécialisé et ses enjeux.

\section{III) La focale du discours spécialisé : entre authenticité et présentisme}

Le souci de réalisme et d'authenticité scientifique n'est pas nouveau dans les séries télévisées médicales. Moser, le créateur de Dr Kildare et de Ben Casey, collaborait déjà avec la Los Angeles County Medical Association dans l'élaboration de ses scénarios. Cependant, l'attention accordée au réalisme scientifique et médical, à une certaine " authenticité ${ }^{23}$ » est allé croissant ces deux dernières décennies. Quoiqu'observable dans de nombreux autres genres, l'attention particulière accordée au réalisme scientifique et médical, qui passe principalement par l'omniprésence du discours médical spécialisé, fait partie intégrante de la dramaturgie du genre et complète les ressorts narratifs plus classiques que sont les codes du soap hospital et ceux de la comédie.

\section{a) Le réalisme médical et scientifique : entre dramaturgie et vulgarisation}

36 Comme l'environnement spécialisé de la médecine sert de trame à l'intrigue, les séries médicales correspondent à la rubrique des Fictions à Substrat Professionnel (FASP), telles que les a définies Michel Petit. Plus précisément, Petit affirme en effet que « c'est parce que le milieu professionnel est la réalité où la fiction se forme en même temps qu'elle le représente qu'il peut être qualifié de substrat ${ }^{24}$. »

Dans les séries médicales comme dans toutes les FASP, il existe deux types d'échanges discursifs, comme le rappelle Petit ${ }^{25}$. On trouve d'une part des dialogues ordinaires sur le quotidien, les problèmes personnels et la vie amoureuse, et d'autre part les échanges entre les spécialistes du domaine médical. Ces dialogues spécialisés peuvent prendre plusieurs formes: le temps du diagnostic médical, le temps de la pratique médicale (pendant une opération au bloc par exemple) et enfin les échanges entre médecins et 
patients. Dans ces différents échanges, le degré de spécialisation de la terminologie varie : elle est beaucoup plus simplifiée et didactique dans le cas de l'échange avec le patient, où la discussion sert des fins de vulgarisation scientifique. La dialectique entre les échanges informels et les échanges spécialisés constitue le nœud narratif des séries médicales, et permet une familiarisation progressive aux contenus présentés.

Les FASP mettent donc un public profane en contact d'un univers et d'un discours spécialisé. Cependant, la notion de "profane» est relative, puisqu'elle est nécessairement complétée par l'expérience des téléspectateurs. Comme le souligne Meyer, la majorité des individus n'a que rarement eu affaire à des policiers et se les représente donc souvent à partir des médias ${ }^{26}$. À l'inverse, la singularité du genre des séries médicales tient au fait que, contrairement aux séries policières et juridiques, la plupart des personnes ont eu à un moment de leur vie une expérience intime avec l'institution biomédicale (consultation, maladie, accident, accouchement, etc...)

\section{b) Des consultants experts de plus en plus présents}

La FASP se caractérise surtout par la collaboration entre scénaristes et experts du domaine, qui contribue au réalisme des univers fictifs proposés. Dans les années 1950 et 1960, l'American Medical Association était sans conteste la source d'expertise la plus influente dans le processus de fabrication du récit et des scénarios, dans un but de préservation de l'autorité professionnelle des médecins, comme nous l'avons vu. À partir des années 1970, la sphère d'influence de l'AMA décline: de nouvelles organisations spécialisées déconstruisent le monopole préexistant de ce lobby.

De nos jours, les sources d'expertise sont multiples. Par exemple, et quoiqu'il n'ait jamais exercé, Michael Crichton, le créateur de la série Urgences était lui-même médecin et s'est inspiré de sa formation pour la série. Les séries Urgences, Grey's Anatomy et $\mathrm{Dr}$ House font régulièrement appel à des consultants extérieurs (médecins, infirmières) pour participer à l'élaboration des scénarios. On peut enfin mentionner le Hollywood, Health and Society Program de l'USC Annenberg Norman Lear Center, un programme universitaire partenaire du Ministère de la santé, qui veille à l'exactitude scientifique et médicale (medical accuracy) des films et des séries traitant de la santé aux États-Unis. Ce programme fournit aux scénaristes des conseils et une documentation à jour, dans un souci de transparence et de sécurité vis-à-vis du public. Depuis sa création en 1994, le Hollywood, Health and Society Program a ainsi collaboré avec de nombreux partenaires. Sur la seule période 2009-2015, l'organisation a travaillé avec plus de 35 chaînes de télévisions ainsi qu'avec les scénaristes de 91 séries télévisées ${ }^{27}$, comme Urgences, Grey's Anatomy, Private Practice ou encore Dr House ${ }^{28}$.

41 Malgré la collaboration d'experts, la fiction embellit parfois la réalité, comme le montre l'exemple de la réanimation cardiaque, acte médical très représenté dans les séries médicales. Comme l'explique le centre national de réanimation des États-Unis dans un article dédié à la question, $68 \%$ des patients survivent à la réanimation dans la série Urgences, contre $46 \%$ dans la série Grey's Anatomy. L'auteur rappelle, statistiques à l'appui, que le taux moyen de réussite des réanimations cardiaques se situe en réalité entre $5 \%$ et $10 \%$.

Comme le montre cet exemple, les inexactitudes médicales sont régulièrement passées au peigne fin par la communauté scientifique. Elles ont même donné naissance à un genre à part entière, celui de la critique de série médicales "en direct " par des 
professionnelles sur certaines chaînes You Tube ${ }^{30}$. Enfin, la recherche universitaire influe parfois sur les contenus, comme l'a souligné Pamela Tytell : les scénaristes de la série Urgences ont corrigé leurs scénarios après que le New England Journal of Medicine leur a demandé de représenter les réanimations cardiaques de manière plus réaliste ${ }^{31}$.

Les séries bénéficient bien entendu d'une licence fictionnelle et l'on ne peut réclamer d'elles qu'elles remplissent une fonction stricte d'information, au même titre que la recherche universitaire et que le documentaire. De ce point de vue, comme le souligne Jean-Marie Schaeffer, la fiction ne vise pas la réalité référentielle ${ }^{32}$. Cela étant, il est notoire que les séries médicales fonctionnent comme un zeitgeist diégétique, et ce indépendamment de l'époque, ce qui nous amène au point suivant.

\section{c) La série hospitalière comme zeitgeist diégétique}

Comme l'expliquent Anne Hudelet et Sophie Vasset, les séries « semblent plus réactives à l'actualité que les films de cinéma, dont la durée de production est plus longue que celle d'un épisode ${ }^{33}$." Ainsi, l'une des forces du discours médical spécialisé est son potentiel d'ancrage dans le quotidien du téléspectateur et les échos qu'il entretient avec lui. En effet, bien que ce dernier ne soit pas spécialiste de santé, les thématiques abordées dans les séries, la solution qu'y proposent les protagonistes et la manière dont les patients y réagissent construisent un pont avec l'actualité et l'expérience du spectateur, qui renforce l'illusion de réalité des contenus sériels.

De ce point de vue, les séries médicales sont empreintes de présentisme, entendu ici au sens de Hartog. Dans son ouvrage Régimes d'historicités, Hartog explique que «l'avenir [s'est mis] à céder du terrain au présent, qui [va] prendre plus de place, jusqu'à sembler depuis peu l'occuper toute entière. On [entre] alors dans un temps de prévalence du point de vue du présent : celui du présentisme justement ${ }^{34}$. » L'artefact sériel est donc une sorte de zeitgeist fictionnel, qui s'inscrit dans les débats médicaux et de santé publique de la société qui les produit à un instant $t$. Ce présentisme est accentué par le modèle du découpage en épisodes propre aux séries, qui confère à la narration une certaine «absence de téléologie ${ }^{35}$ » ainsi que par la grammaire audiovisuelle, les choix de réalisation et ceux de post-production. Pour ne citer qu'un exemple, le réalisme de la série Urgences est renforcé par le tournage en steadicam ainsi que par l'utilisation fréquente de plans séquences, qui font « correspondre le temps réel et le temps filmé, le cadre de réception et le cadre d'émission ${ }^{36}$ ", comme le souligne Glevarec. Enfin, le présentisme est central à l'économie narrative des séries médicales: sa forte valeur dramatique en fait un auxiliaire précieux de l'illusion fictionnelle et de la «suspension volontaire de l'incrédulité » du spectateur (willing suspension of disbelief), pour reprendre l'expression de Samuel Taylor Coleridge ${ }^{37}$.

Le genre sériel médical a subi de profondes mutations au cours du demi-siècle dernier. La représentation de l'institution biomédicale s'est transformée, nuancée et complexifiée pour aller vers davantage de réalisme, qu'il s'agisse de la manière dont les médecins sont dépeints, des valeurs métaphoriques et allégoriques que l'on peut attribuer à l'hôpital ou encore de la qualité scientifique des contenus spécialisés présentés au public. En dépit de ces évolutions notoires, et bien que les représentations 
de l'institution biomédicales se soient pour partie émancipées du carcan que leur imposait l'AMA au milieu du $\mathrm{xx}^{\mathrm{e}}$ siècle, les séries télévisées médicales se sont progressivement érigées en canon caractérisé par une "asymétrie réaliste ». Plus précisément, l'ensemble des choix de focalisation qui régissent et assurent le succès commercial des séries médicales (focale sur les médecins, sur l'hôpital urbain et sur le discours médical spécialisé) participent d'une hypervisibilisation de la science médicale et de ses agents, cadre qui opère simultanément comme un cache des réalités socioéconomiques de la société étatsunienne qu'il entend représenter. La deuxième partie de cet article étudie les enjeux épistémiques de cette "asymétrie réaliste », en étudiant tout ce que ce cadre laisse hors cadre et hors champ.

\section{IV) Le canon sériel médical : un cache}

Parce qu'il est un cadre, le canon sériel médical étatsunien fonctionne aussi comme un cache de la réalité socio-économique dans l'arène de la santé. Pour un observateur de la vie sociale de ce pays, l'invisibilisation des inégalités d'accès aux soins médicaux dans les séries médicales est frappante. Cette invisibilisation fait partie de l'économie narrative des séries hospitalières et constitue l'autre facette de l'asymétrie réaliste décrite en introduction, et que nous analysons plus avant dans les sections qui suivent.

\section{a) Les médecins du canon sériel : un portrait tronqué ?}

On l'a vu, les protagonistes des séries médicales étatsuniennes sont aujourd'hui plus divers qu'à tout autre moment de l'histoire de ce canon culturel. En dépit de ces évolutions, qui reflètent des transformations tangibles au sein de la société, on constate en parallèle une permanence des choix de focalisation dans la représentation des praticiens et celle de leur activité. La permanence de ces choix doit être étudiée pour mieux en révéler les enjeux épistémiques.

En effet, les séries médicales offrent un reflet incomplet et romancé de la pratique médicale. Malgré l'introduction de nuances, les séries médicales récentes participent à l'ossification du genre. En effet, les héros des séries les plus populaires comme Urgences et Grey's Anatomy sont invariablement spécialistes, presque toujours chirurgiens. La focalisation sur la chirurgie s'explique par son fort potentiel dramatique, avec lequel une série qui suivrait par exemple les difficultés des médecins travaillant dans un community health center $^{38}$ aurait peine à rivaliser. Par ailleurs, le succès limité de nouveaux programmes traitant de la médecine libérale, comme Private Practice (spin-off de Grey's Anatomy) atteste de la difficulté d'innover dans la dramaturgie du genre.

Outre la focalisation sur les spécialités construites comme socialement prestigieuses, ces séries continuent de véhiculer une image idéalisée de la relation médecin-patient, bien éloignée de la réalité. En effet, et contrairement à ce que l'on observe dans les séries, on estime que les médecins passent un quart de leur temps au chevet des patients, et le reste à traiter de tâches administratives, en particulier à négocier avec les compagnies d'assurance et l'industrie pharmaceutique dans le cas étatsunien. Lorsqu'elles sont abordées (ce qui est rare), les négociations avec les compagnies d'assurance maladie sont présentées comme un épiphénomène. La série Dr House constitue sans doute l'exemple le plus frappant de cette invisibilisation. En effet, ce programme n'aborde jamais la question des coûts de santé ni celle de l'assurance 
maladie ${ }^{39}$. Gregory House semble bénéficier d'un budget illimité pour pratiquer son métier. En réalité, depuis les années 1970, l'application d'une logique gestionnaire et commerciale à l'hôpital fait que les médecins sont pénalisés financièrement s'ils dépassent les budgets qui leurs sont alloués pour chaque type d'acte ou chaque pathologie. Dans le cas spécifique de Dr House, l'invisibilisation du financement des soins est d'autant plus étonnante que le traitement des maladies rares et des maladies orphelines n'est presque jamais couvert par les assurances. Dans cette série, l'occultation des coûts médicaux se fait à la faveur d'une focalisation sur le monopole du diagnostic et de la définition des maladies dont jouissent les médecins, House se déclarant lui-même " diagnosticien » (compétence en théorie propre à tout médecin).

51 En somme, la clef de voûte du genre et les clefs de son succès à travers les décennies demeurent inchangés: les séries contemporaines capitalisent toujours sur l'aura culturelle des médecins, renforcent la fascination thaumaturgique des téléspectateurs, et alimentent consciemment ou non leur croyance dans le miracle médical. Le statut iconique du médecin dans la société est d'ailleurs abordé de manière amusante dans d'autres séries comme Friends. Dans ce programme, l'une des plaisanteries récurrentes concerne le titre de docteur que détient Ross, l'un des personnages principaux. Ross est effectivement docteur en paléontologie, ce qui lui vaut le sarcasme des autres personnages, qui ne manquent pas de lui rappeler qu'il n'est pas un «vrai» docteur (c'est-à-dire docteur en médicine), et donc parfaitement inutile à la société.

En outre, dans les séries récentes (et contrairement à celles du passé), la relation entre médecin et patient sert souvent de prétexte aux intrigues amoureuses et personnelles de l'équipe médicale. Pour ne citer qu'un exemple, voici un extrait de l'échange entre le docteur Shepherd et sa femme Addison dans un épisode de Grey's Anatomy (saison 02, épisode 03). Alors qu'ils évaluent les chances de survie d'un bébé prématuré, la discussion dérive sur l'infidélité d'Addison, que Derek lui reproche toujours, sous le regard gêné de l'interne Izzie Stevens, témoin de la scène :

DR DEREK SHEPHERD. Elle a des méningites à répétition. Sa vie sera courte et pénible.

DR ADDISON SHEPHERD. Ça, tu n'en sais rien.

DR DEREK SHEPHERD. C'est mon métier de savoir ce genre de choses.

DR ADDISON SHEPHERD. Tu n'es pas dieu Derek.

DR DEREK SHEPHERD. Qu'est-ce que tu viens de dire?

DR ADDISON SHEPHERD. Désolée chéri mais c'est vrai. Il ne t'appartient pas de décider... DR DEREK SHEPHERD. Je rêve ou tu viens de me dire chéri ? Ne m'appelle pas chéri.

DR ADDISON SHEPHERD. Vous n'êtes pas dieu Dr Shepherd. Si un patient a la moindre chance de survivre, et je pense que c'est son cas à elle, et bien c'est ta responsabilité de...

DR DEREK SHEPHERD. Ne viens pas me parler de responsabilité.

DR ADDISON SHEPHERD. Tu as prêté serment, Derek.

DR DEREK SHEPHERD. Tu es mal placée pour me parler de serments.

DR ADDISON SHEPHERD. Derek, j'ai fait une erreur. Tout le monde fait des erreurs.

DR DEREK SHEPHERD. Tu as couché avec mon meilleur ami dans mes draps préférés.

DR ADDISON SHEPHERD. Les draps en flanelle ? Mais tu détestes ces draps...

DR DEREK SHEPHERD. Ah non, je les adore.

DR ADDISON SHEPHERD. C'est comme les draps italiens avec...

DR DEREK SHEPHERD. Arrête de me parler de draps.

INTERNE IZZIE STEVENS. Heu, désolée, je vais y aller. Je vais regarder les résultats des

tests.

DR DEREK SHEPHERD. Addison, arrête.

DR ADDISON SHEPHERD. Mais enfin c'est une battante, regarde ! Elle a tenu jusqu'ici... 
DR DEREK SHEPHERD. Ne t'attache pas. Ça ne te regarde pas.

53 L'entre-soi médical constitue la focale et le cadre de l'intrigue; les questions socioéconomiques sont reléguées au hors-champ sériel. Il convient cependant de préciser que certaines séries ont pris leurs distances d'avec ces représentations, en particulier les séries médicales à la marge du canon comme Dr House. Dans Dr House, la satire du canon sériel médical transparaît grâce à la mise en abîme. En effet, Gregory House est un téléspectateur assidu de Prescription Passion, série dans la série qui apparaît dès le pilote et qui parodie les codes et les conventions de l'hospital soap. Dans Prescription Passion, le docteur Brock Sterling, Dom Juan notoire, entame une liaison avec la sœur de sa fiancée, qui tombe accidentellement enceinte de lui. Brock Sterling est également alcoolique, boit des gin tonic avant d'opérer, affirmant que ces cocktails lui permettent de mieux travailler. La satire de la mise en abîme est poussée à l'extrême dans l'épisode «Living the Dream» (saison 4, épisode 14), dans lequel Gregory House soigne Evan Greer, la star qui incarne le Dr Brock Sterling sur le petit écran. Greer explique qu'il déteste son travail en dépit de l'argent et de la notoriété, que les intrigues du feuilleton sont ridicules, que les acteurs sont médiocres et qu'il aimerait consacrer son temps et son énergie à quelque chose de plus gratifiant. Le propos de Greer a vocation satirique, c'est-à-dire précisément à faire réfléchir sur les représentations, en particulier à celle du métier de médecin et de son statut iconique dans la société.

\section{b) L'hôpital du canon sériel : stratégie d'évitement des inégalités en santé?} États-Unis bien qu'il en soit une institution centrale. Les patients s'y rendent quand ils sont atteints de maladies longues (cancer) et en cas d'urgence. Ensuite, des séries comme Urgences et Grey's Anatomy donnent à voir des problèmes sociaux existants : Urgences fut la première à mettre en scène un patient atteint du sida. Des sujets comme l'addiction aux drogues dures, les maladies sexuellement transmissibles, l'euthanasie, etc. sont régulièrement abordés dans les séries contemporaines. Enfin, même si des maladies rares ou spectaculaires sont légions dans les séries médicales et servent une fonction de dramatisation, les trois pathologies les plus représentées dans des programmes comme Urgences ou Grey's Anatomy sont les blessures, les maladies cardiovasculaires et le cancer ${ }^{40}$, un triptyque qui correspond à l'état de santé global de la population étatsunienne.

En outre, la représentation de la réalité sociale et financière des patients est limitée dans ces séries. Tout d'abord, bien que les représentations des médecins soient aujourd'hui beaucoup plus variées que par le passé, celle des patients, elle, est demeurée globalement monolithique. Plusieurs études ont montré que le profil démographique des patients représentés dans les séries comme Urgences et Grey's Anatomy est loin de refléter la réalité. Le patient-type de ces séries est un homme blanc d'une quarantaine d'années. Les patients noirs, hispaniques, les patientes, les seniors et les enfants sont globalement sous-représentés ${ }^{41}$, alors même que ce sont eux qui

TV/Series, $17 \mid 2020$ 
fréquentent majoritairement les hôpitaux publics et les services des urgences en particulier.

De plus, la représentation de la prise en charge financière des patients est souvent passée sous silence. Dans les séries plus anciennes comme Dr Kildare, le thème de l'assurance maladie est mentionné et intégré à la trame narrative de manière discrète. Par exemple, dans le pilote de la série, James Kildare reçoit une patiente et lui demande si elle est couverte, ce à quoi elle répond oui. Dans le sixième épisode de la première saison, un patient peste contre l'équipe médicale :

PATIENT. Je paie cher pour être ici, donc je veux être très bien soigné !

DR KILDARE. Je signe ses papiers de décharge. Je pense qu'il veut prendre des vacances grâce à son assurance maladie.

Dans cet extrait, ce n'est pas le fait que le patient ait ou non une assurance maladie qui pose problème, mais bien le fait qu'il puisse utiliser cette protection à mauvais escient (c'est-à-dire pour ne pas travailler), un constat conforme au ton moralisateur des séries produites à cette époque. Inversement, dans les séries plus récentes, l'invisibilité des débats sur la couverture santé est marquante, non seulement parce qu'il n'existe pas de droit à la santé afférent à la citoyenneté aux États-Unis, mais aussi parce que cette thématique était un enjeu omniprésent dans les débats politiques des années Clinton (1992-2000) jusqu'aux années Obama (2008-2016).

Ainsi, de manière surprenante, le téléspectateur qui regarde les séries médicales actuelles peut conclure que tout un chacun peut être pris en charge gratuitement et sans contrepartie à l'hôpital, ce qui est loin d'être vrai. Bien sûr, et comme expliqué plus haut, les urgences n'ont plus le droit de refuser des soins aux patients en état grave, conformément à la loi EMTALA de 1986. Cependant, la loi stipule également que le seul devoir des urgentistes est de stabiliser le patient, non de veiller à la poursuite des soins et à leur financement jusqu'à sa sortie. Il est vrai que dans la réalité, les médecins pratiquent des soins gratuitement après la mise hors de danger du malade ${ }^{42}$, mais jamais dans les proportions qui sont celles des séries télévisées. La focalisation sur l'hôpital et les urgences hospitalières pourrait donner le sentiment que l'accès aux soins médicaux est un droit aux États-Unis alors qu'il n'en est rien. La question de l'assurance maladie est abordée dans les séries médicales bien sûr, mais celle-ci fait l'objet d'un traitement marginal et surtout ambigu. D'une part, les inégalités inhérentes au système de santé étatsunien sont l'un des ressorts de la dramaturgie des séries médicales, qui les exploitent pour mettre en relief le danger qui pèse sur les patients et surtout le courage et l'abnégation des médecins. D'autre part, le problème de l'accès aux soins pour les patients et celui leur financement est souvent neutralisé dans les scénarios. Les quatre exemples ci-après, tirés de Grey's Anatomy illustrent cette double dynamique.

Dans «The Self-Destruct Button» (saison 1, épisode 7), un couple s'inquiète du montant des soins que l'hôpital Seattle Grace leur propose pour le traitement de leur fille Jamie :

MARI. Nous travaillons tous les deux, et je ne sais pas si notre assurance...

FEMME. On sait que cela peut coûter très cher...

DR SHEPHERD. Je ne veux pas que vous vous souciiez de ça.

61 Dans «Raindrops Keep Falling on my Head» (saison 2, épisode 1), Joe, le barman de l'Emerald City Bar est victime d'un anévrisme. Il discute avec le Dr Shepherd de la procédure, et demande le coût des soins :

JOE. Combien? Combien est-ce qu'un truc comme ça coûte? 
DR SHEPHERD. Je ne pense pas que vous devriez vous soucier de ça maintenant.

JOE. Ok. Vous me dites que vous pouvez me tuer et me ramener à la vie, et je vous crois. Vous êtes médecin. Mais je tiens un bar. Je n'ai pas d'assurance maladie, du coup je me soucie moins de l'opération que de ce que je vais faire quand j'y aurai survécu. J'ai besoin d'un chiffre. Dix mille? Vingt mille? Trente mille?

DR SHEPHERD. C'est au moins deux cent mille dollars.

Dans «The Ties that Bind» (saison 5, épisode 8), le Dr Shepherd discute avec un patient sans-abri et lui explique :

DR SHEPHERD. Il y a un centre de désintoxication pas très loin d'ici. Medicare ${ }^{43}$ s'en occupe. Vous pouvez y rester. Vous aurez à manger. Un lit. Vous pourrez y rester aussi longtemps que vous voulez.

Enfin, dans «Adrift and at Peace» (saison 7, épisode 10), le Dr Teddy Altman propose à l'un de ses patients, Henry, atteint d'une tumeur et sans assurance maladie, de se marier avec elle pour qu'il puisse bénéficier de sa couverture maladie, ce qu'il finit par accepter.

En somme, la question de l'inégalité d'accès aux soins médicaux n'est pas totalement passée sous silence dans ces séries, cependant, son traitement ambigu contribue à la rendre marginale: dans les exemples précités, le docteur Shepherd interrompt régulièrement les patients inquiets de l'impact financier de leur prise en charge médicale, comme si cette question était subsidiaire. Ainsi, de manière paradoxale, le gros plan sur les urgences hospitalières fonctionne comme une stratégie d'évitement des inégalités plutôt que comme confrontation à leur réalité. Ceci importe d'autant plus que dans le contexte spécifique de réception étatsunien, les résonnances de ce thème sont particulièrement forts : la peur de perdre son assurance maladie (ou le fait d'en être dépourvu) rend les sujets comme la maladie et la mort beaucoup plus complexes, car le coût des soins médicaux demeure la première cause de ruine financière personnelle dans ce pays, même dans le cas où la personne est assurée ${ }^{44}$.

Notons toutefois que certaines séries comme Scrubs offrent une lecture plus complète des problèmes d'accès aux soins aux États-Unis. Dans cette série, le cadre médical est souvent subverti : il sert de prétexte à la critique de la société et à celle du système de santé, ce qui est suffisamment rare dans le panorama contemporain des séries médicales pour être souligné. Par exemple, dans l'épisode 13 de la saison 6, une patiente arrive à l'hôpital et le principal protagoniste, le docteur J.D., explique en voix off comment il traite le cas des patients sans assurance maladie :

INFIRMIÈRE CARLA ESPINOSA : Nom et assurance, s'il-vous-plaît?

PATIENTE MAGGIE KENT. Maggie Kent. Pas d'assurance.

INFIRMIÈRE CARLA ESPINOSA : Bon. On va vous trouver une chambre.

VOIX OFF DE J.D. Quand un patient n'a pas d'assurance maladie, il faut contourner le système. D'abord, il faut trouver quelqu'un qui vient de mourir.

DOCTEUR J.D. Super! M. Rabinowitz vient de crever! (regard interloqué de la patiente). Oh ne vous inquiétez pas. Il a mis son engin dans une prise électrique. Il ne faut pas faire ça.

VOIX OFF DE J.D. Ensuite il faut convaincre le type qui bosse à la morgue de ne pas trop être regardant sur la paperasse pour que le lit du patient mort reste disponible. (On voit J.D. corrompre l'infirmier qui s'occupe de la morgue en lui donnant des sucettes). Ensuite, il faut se mettre les infirmières dans la poche.

INFIRMIÈRE. (apporte un plateau repas à la patiente Maggie Kent et dit) Bon appétit M. Rabinowitz!

VOIX OFF DE J.D. Ensuite il faut trouver un chirurgien qui accepte de faire une opération gratuitement.[...] Mais surtout, il faut s'assurer qu'aucun de vos 
supérieurs ne découvre qu'un des patients n'a pas d'assurance maladie, même si ce sont eux qui ont admis le patient à l'hôpital au départ.

PATIENTE MAGGIE KENT. (au docteur Kelso qui l'a admise à l'hôpital) Tout ce que je veux c'est vous remercier de faire tout cela même si je n'ai pas d'ass...(elle est interrompue par les médecins J.D. et Turk, qui demandent à parler à Kelso d'urgence).

Dans cet extrait, l'inégalité d'accès aux soins médicaux sert de ressort comique, cependant le parti pris est satirique car il permet de montrer deux médecins, J.D. et Turk, cherchant à honorer coûte que coûte leur serment d'Hippocrate. On retrouve ce type de scénarios dans d'autres séries comiques, qui s'attaquent parfois plus volontiers au sujet de l'accès à la santé que ne le font les séries médicales. Par exemple, dans la saison 1 de Friends, Rachel et Monica vont à l'hôpital car Rachel s'est foulé la cheville. Rachel n'a pas d'assurance maladie et se fait passer pour Monica pour bénéficier des soins. Le comique de situation est accentué par le fait que le médecin en charge de la consultation aux urgences n'est autre que George Clooney. Ce caméo est un clin d'œil à la série Urgences (diffusée à la même époque) ainsi qu'à la television literacy du téléspectateur. Dans un autre épisode (saison 6, épisode 4), Joey, qui est acteur, apprend dans un courrier que son assurance maladie est résiliée car il n'a pas travaillé suffisamment l'année précédente. Déstabilisé par cette nouvelle, il explique à son colocataire Chandler :

JOEY. C'est nul! Quand j'avais une assurance maladie, je pouvais me faire renverser par un bus ou prendre feu, tu vois! Et c'était pas grave. Maintenant il faut que je fasse gaffe ${ }^{45}$ ?

Pour éviter la ruine financière, Joey décide de se mettre au sport mais se fait une hernie. Au cours de cet épisode, il parvient à tourner dans une publicité où, immobilisé, il joue un père mourant qui parle à son fils, touche un cachet et retrouve son assurance maladie. Dans ces exemples comme dans celui de Scrubs, l'inégalité d'accès à la santé est utilisée comme ressort comique. Dans d'autres séries comme Breaking Bad, elle sert de ressort dramatique à toute la série. Le personnage principal de la série, Walter White, un homme blanc, la quarantaine, professeur de chimie dans un lycée public, décide de produire et de vendre de la méthamphétamine pour payer sa chimiothérapie. Son alter ego sociopathe Heisenberg (le nom de dealer qu'il se choisit) est l'incarnation métaphorique du désespoir auxquels certains Américains sont confrontés dans un système où la protection maladie est limitée et conditionnelle. White bénéficie pourtant d'une assurance maladie (comme tous les salariés du secteur public), mais la peur d'une mort prématurée le pousse lui et sa famille à faire le choix d'être traité par le Dr Delcavoli, oncologue superstar qui n'est pas partenaire du réseau de soins coordonnés (Health Maintenance Organization, HMO) auquel White est affilié. Breaking Bad rappelle combien les Américains sont vulnérables face à la maladie, même lorsqu'ils travaillent et sont couverts par une assurance maladie standard ${ }^{46}$. Une image valant souvent plus que tous les mots, le meme ci-dessous, qui compare le système de santé étatsunien à celui de son voisin le Canada (où l'assurance maladie est universelle), résume de manière synthétique et humoristique que les inégalités d'accès aux soins médicaux sont l'un des principaux ressorts scénaristiques dans le contexte étatsunien : 
Meme internet montrant la transposition de l'intrigue de Breaking Bad dans le contexte canadien.

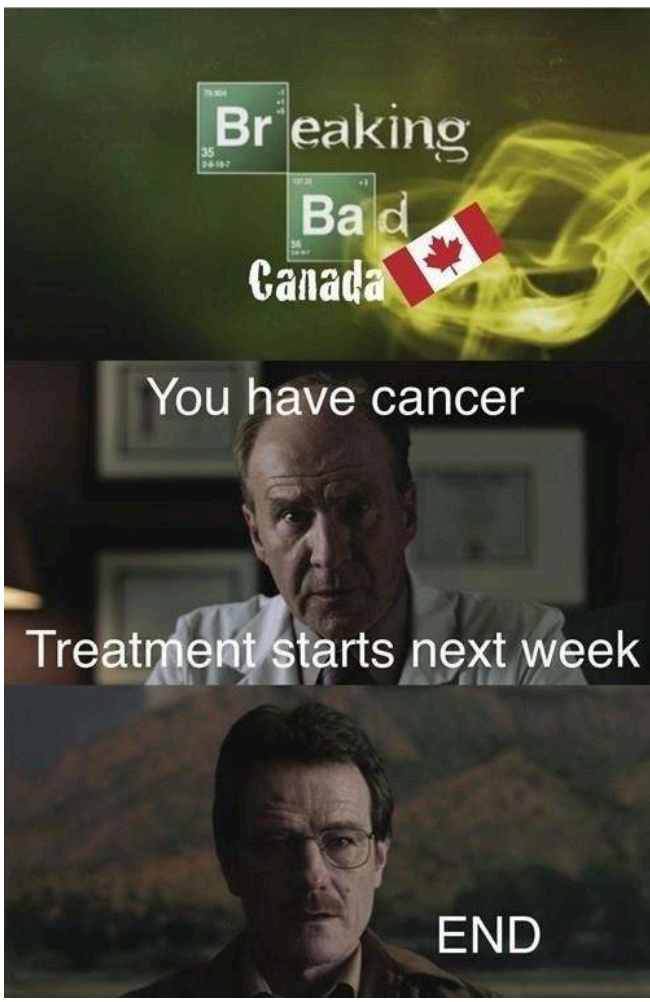

Source : https://cheezburger.com/7462907392

\section{c) Le présentisme des séries télévisées médicales : cache des inégalités?}

Il convient en dernier lieu analyser les enjeux de la focalisation discursive dans les séries médicales. Il ne s'agit pas ici de traiter de la question de l'interprétation de la fiction, car, on le sait, celle-ci n'est jamais univoque. Il s'agit plutôt de réfléchir à la puissance sémiotique de l'artefact série, objet construit, non neutre, transmettant une " image-monde ${ }^{47}$ " qui va façonner les représentations des téléspectateurs, dans ce cas précis, des représentations au sujet de l'institution biomédicale.

Premièrement, la puissance sémiotique des séries, c'est-à-dire la force des représentations et des messages qu'elle diffuse, est due au médium: en tant que " storyteller » principal dans la société étatsunienne contemporaine ${ }^{48}$, la télévision est détentrice d'un fort pouvoir de cadrage sur les représentations. La télévision est en outre un médium unidirectionnel, qui présuppose une certaine passivité de la part du spectateur. Enfin, le temps passé devant la télévision peut rendre les téléspectateurs perméables aux représentations qu'elle diffuse ${ }^{49}$, quoique ceux-ci ne soient bien évidemment pas dupes.

Deuxièmement, la puissance sémiotique des séries tient à la renaissance du genre ces deux dernières décennies. Les séries télévisées, «boudées naguère par l'intelligentsia, considérées à l'origine comme un genre mineur, un sous-produit culturel voué au seul divertissement, [...] ont aujourd'hui conquis leurs lettres de noblesse ${ }^{50}$.» 
71 Troisièmement et dernièrement, la puissance sémiotique des séries s'inscrit dans un contexte économique de production bien précis. Parce que les séries sont une marchandise culturelle, l'écriture, le rythme du récit et les thèmes qui y sont abordés suivent une logique financière et commerciale ${ }^{51}$. Réussir la course à l'audience dans un paysage médiatique très concurrentiel nécessite souvent de recycler les recettes existantes : dans le cas des séries médicales, il s'agit de perpétuer le canon du genre et son asymétrie réaliste, car les focales choisies et les sujets qui y sont abordés sont spectaculaires (au sens propre du terme) et populaires.

72 Ces trois forces expliquent la puissance sémiotique des séries et contribuent à expliquer qu'elles puissent servir de "réservoir de références ${ }^{52}$ ", c'est-à-dire de grilles de lecture, d'analyses et de réactions, de « modèles de comportements ${ }^{53}$ " mobilisables par les téléspectateurs au quotidien. Les enjeux liés à l'assimilation des représentations sont considérables car le schéma narratif des séries favorise l'immersion, la familiarisation, et l'observation de ressemblances entre la réalité sociale vécue et celle qui est figurée dans les contenus sériels ${ }^{54}$. Les enjeux liés à l'assimilation des représentations importent aussi en ce que les médias de masse déforment les perceptions de la réalité (phénomène que les auteurs de fiction n'anticipent pas forcément par ailleurs), comme l'a montré le théoricien de la cultivation theory George Gerbner $^{55}$. De manière plus localisée, l'assimilation de représentations fictives au sujet de l'institution biomédicale importe dans le sens où les Américains sont plus exposés aux médecins fictifs qu'ils ne le sont à de vrais médecins, car « le téléspectateur moyen voit quelque douze docteurs et six infirmier.es chaque semaine dans les émissions prime time, dont trois docteurs et un.e infirmier.e dans des rôles de premier plan ${ }^{56}$. "

73 C'est dans ce contexte que les enjeux de la focalisation discursive des séries médicales étatsuniennes, que nous avons nommé «asymétrie réaliste ", apparaissent plus clairement. Le présentisme du métadiscours sur l'institution biomédicale dans les séries médicales produites aux États-Unis est l'un des traits les plus nets de cette asymétrie réaliste: les questions politiques et économiques (difficulté de l'accès aux soins médicaux, montant faramineux de la prise en charge financière, etc) sont presque toujours écartées au profit de choix de focalisation du hic et nunc, adaptés à la focalisation sur les urgences hospitalières et à leur fort potentiel dramatique. Quoique sans valeur absolue, ce commentaire glané sur un site d'analyse des taux d'audience de la série Grey's Anatomy est parlant à plus d'un titre. Un utilisateur du site écrit : « Est-ce que quelqu'un a remarqué ce qui s'était passé ? Dès que la série a abordé des questions politiques, les taux d'audience ont chuté ${ }^{57}$. »

74 La focalisation présentiste de l'asymétrie réaliste, en apparence neutre et a-politique, reflète avant tout les impératifs du divertissement et de la viabilité financière, propre au genre sériel. Cependant, cette asymétrie réaliste est un miroir déformant: par son prisme, on voit un système de santé fonctionnel, généreux et universel, où chacun peut être soigné sans se soucier du coût faramineux des soins qui lui sont prodigués. Cette asymétrie réaliste véhicule également une image valorisante des médecins tout à leur mission de soignant, et sur qui les injonctions de contrôle des coûts ne semblent pas peser. Ce métadiscours est problématique car il ne prend pas acte de l'accroissement spectaculaire des inégalités aux États-Unis en matière de santé. Il est conservateur en ce qu'il nourrit la chimère d'un contrat social égalitaire aux États-Unis. 


\section{Conclusion} équipe de médecins chevronnés, cadre fictif de l'hôpital urban et centralité d'un discours scientifique ancré dans le présent) ont contribué à la consolidation d'un canon qui est à la fois un cadre et un cache, et dont la dialectique permet de mesurer le progrès médical et l'entrée de nouveaux profils de praticien dans le champ, mais ne rend pas aussi fidèlement compte du recul de la justice sociale dans ce pays.

79 Ces choix de cadrage résultent bien entendu de l'impératif d'économie narrative propre aux séries médicales, de la logique financière qui façonne le contour des scénarios et les thèmes qui y sont abordés. Il est donc sans doute naturel que dans la fiction le divertissement prenne le pas sur la véracité des contenus.

80 Malgré tout, on constate que le « retour du même » dans les séries médicales est coupé des débats politiques qui lui sont contemporains. Le présentisme des séries médicales est donc lui aussi asymétrique : il ne reflète que partiellement la réalité du temps présent. La plupart des séries médicales relèguent les débats sur le financement des soins ainsi que les débats politiques portant sur la santé au hors-champ, et l'avenir de Medicare, de Medicaid et de l'obamacare sont passés sous silence, alors même qu'ils saturent l'espace médiatique non fictionnel.

81 Pour conclure, on soulignera néanmoins un atout majeur des séries télévisées hospitalières, à savoir que l'invisibilisation relative des inégalités - notamment financières - dans le domaine de l'accès aux soins, offre un contrepoint à la fiscalisation omniprésente du débat sur les politiques sociales aux États-Unis et ailleurs dans le monde. En effet, au sein de l'univers fictif des séries médicales, la question des coûts, pourtant centrale dans un pays où la santé est un privilège onéreux, est reléguée au hors champ ou neutralisée. Il est probable que cette invisibilisation ne serve pas uniquement les multiples impératifs d'une narration dédiée au suspense et à l'émotion : elle peut également être lue comme une prise de position, une proposition fictionnelle de la part des scénaristes (quoique souvent discrète), une feuille de route morale sur la hiérarchie des priorités qui devrait être adoptée dans un tel système, à savoir que la santé du patient et la vie humaine devraient primer sur les logiques économiques et financières qui régissent le fonctionnement des hôpitaux dans ce pays. 


\section{NOTES}

1. Marshall MacLuhan, Understanding Media : The Extension of Man, London, Sphere Books Limited, 1967, p. 351.

2. Michel Petit, «Quelques réflexions sur la fiction à substrat professionnel : du général au particulier ", Aspects de la fiction à substrat professionnel, Petit, Michel et Isani, Shaeda (dir.), coll. Travaux, Bordeaux, vol. 20, 2004, p. 14 [p. 3-23].

3. Voir par exemple Elodie Vargas, «Les séries télévisuelles 'scientifiques' médicales : quelle scénographie des sciences et des techniques? ", Les Enjeux de l'information et de la communication, $\mathrm{n}^{\circ} 16,2015$, p.127-141; Jean-Pierre Charpy, «La FASP médicale comme outil pédagogique: authenticité des textes ou altération de l'authenticité ? ", Cahiers de l'APLIUT, vol. 30, n², 2011, p. 65-81.

4. Pamela Tytell, " "Is there a Doctor in the HOUSE ?" : les séries télévisées médicales face au monde réel ",TV/Series [en ligne], vol.1, 2012, mis en ligne le 15 mai 2012, http:// journals.openedition.org/tvseries/1551 (consulté le $1^{\text {er }}$ octobre 2018); Jean Peneff, "La face cachée d'Urgences. Le feuilleton de la télévision ", Genèses, vol. 30, 1998, p.122-145; Sabine Chalvon-Demersay, "La Confusion des Conditions : une enquête sur la série télévisée Urgences » Réseaux, 95, 1999, p. 235-283 ; Hervé Glevarec, «Trouble dans la fiction. Effets de réel dans les séries télévisées contemporaines et post-télévision ", Questions de communication, vol. 18, 2010, p. 214-238.

5. François Hartog, Régimes d'historicité, présentisme et expérience du temps, Paris, Éditions du Seuil, 2003, p. 108.

6. André Bazin, Qu'est-ce que le cinéma ? Le cinéma et les autres arts, Paris, Éditions du Cerf, 1959, p. 100.

7. Sarah Sepulchre, «Fictions de guerres", Médiamorphoses, hors-série n³, 2007, p. 103 [p. 102-105].

8. Jean-Marie Schaeffer, « Du texte au genre. Notes sur la problématique générique », Théorie des genres, ed. G. Genette G. et al., Paris, Le Seuil, 1986, p. 197 [p. 179-205].

9. Mary Ann Watson, The Expanding Vista: American Television in the Kennedy Years, Durham, Duke University Press, 1994, p. 8.

10. Joseph Turow, Playing Doctor: Television, Storytelling and Medical Power, Ann Arbor, University of Michigan Press, 2010.

11. Grey's Anatomy, «The Self-Destruct Button » (saison 1, épisode 7), traduction de l'auteure : « OK. Anyone who says you can sleep when you die, tell them to come talk to me after a few months as an intern. Of course, it's not just the job that keeps us up all night. I mean, if life's so hard already, why do we bring more trouble down on ourselves? What's up with the need to hit the self-destruct button? Maybe we like the pain. Maybe we're wired that way. Because without it, I don't know, maybe we just wouldn't feel real. What's that saying? Why do I keep hitting myself with a hammer? Because it feels so good when I stop."

12. Keith Wailoo, «Revisiting the Role of Science in the Construction and Erosion of Medical Dominance ", Journal of Health Politics, Policy and Law, vol. 29, nº4-5, 2004, p. 643-659.

13. Elisabeth Fauquert, « Du pareil au même ? Figures du patient américain du New Deal au Patient Protection and Affordable Care Act (2010) », Anthropologie \& Santé [en ligne], vol. 8, 2014, http:// journals.openedition.org/anthropologiesante/1383 (consulté le 12 août 2018).

14. Sabine Chalvon-Demersay, "La confusion des conditions. Une enquête sur la série télévisée Urgences », Réseaux, vol. 17, n95, 1999, p. 241 [p. 235-283]. 
15. Pamela Tytell, «Is there a Doctor in the HOUSE ? " : les séries télévisées médicales face au monde réel »,TV/Series 1 |2012, mis en ligne le 15 mai 2012, consulté le 29 octobre 2018. URL : http://journals.openedition.org/tvseries/1551.

16. Jason Jacobs, Body Trauma: New Television Medical Dramas, London, BFI, 2003, p. 94.

17. François Jost, De quoi les séries télévisées américaines sont-elles le symptôme? coll. Débats, Paris, CNRS éditions, 2011, p. 16.

18. Ibid.

19. Nicolas Boileau, L'Art poétique, Chant III (vers 45-46), Bruxelles, Imprimerie Royale (1817), p. 34 .

20. Cet acronyme signifie « Mobile Army Surgical Hospital ».

21. Elisabeth Fauquert, op. cit.

22. Patrick Lecomte, Communication, Télévision et Démocratie, Lyon, Presses Universitaires de Lyon, 1993, p. 32.

23. Sarah Sépulchre, « Fictions de guerres », op. cit.

24. Michel Petit, «Quelques réflexions sur la fiction à substrat professionnel », op. cit.

25. Ibid.

26. Michaël Meyer, «Introduction. Le policier face à son double médiatique : pistes et enjeux pour l'analyse de la nouvelle visibilité policière", in Médiatiser la police, policer les médias, ed. M. Meyer, Lausanne, Antipodes, 2012, p. 5-20.

27. Site de l'Hollywood Health and Society Program, https://hollywoodhealthandsociety.org/ about-us/history-hhs-numbers (consulté le 4 septembre 2018).

28. Ibid.

29. National CPR Association, « Common Medical Inaccuracies in Medical Dramas », 21 mars 2017, https://www.nationalcprassociation.com/common-medical-inaccuracies-in-medical-dramas (consulté le 04 septembre 2018).

30. On pense ici à « Doctor Mike ", chaîne You Tube suivie par plus de 2 millions d'abonnés, dans laquelle Mike Varshavski, un jeune docteur séduisant donne des conseils aux internautes et consacre plusieurs épisodes à "réagir » à des scènes de séries médicales et à les commenter comme Scrubs, Dr House ou Grey's Anatomy. Lien vers la chaîne You Tube: https:// www.youtube.com/channel/UC0QHWhjbe5fGJEPz3sVb6nw (consulté le 5 septembre 2018).

31. Pamela Tytell, «Is there a Doctor in the HOUSE ? ", op. cit.

32. Jean-Marie Schaeffer, «Quelles vérités pour quelles fictions? ", L'Homme, n¹75-176, 2005, p. 19-36.

33. Ariane Hudelet et Sophie Vasset, «Préface ", TV/Series [En ligne], $1 \mid 2012$, mis en ligne le 15 mai 2012, http://journals.openedition.org/tvseries/1035 (consulté le $1^{\text {er }}$ novembre 2018).

34. François Hartog, Régimes d'historicité, op. cit., p. 108.

35. Hervé Glevarec, La sériephilie. Sociologie d'un attachement culturel, Paris, Ellipses, 2012, p. 88.

36. Hervé Glevarec, « Troubles dans la fiction », op. cit.

37. Samuel Taylor Coleridge, Biographia Literaria, Londres, The Floating Press, 2009, p. 239.

38. Les community health centers sont des cliniques de proximité proposant des soins médicaux aux résidents les plus pauvres.

39. À l'exception notoire de l'épisode 19 de la sixième saison, qui montre les négociations houleuses entre les compagnies d'assurance et le Princeton-Plainsboro Teaching Hospital, où House exerce.

40. Yinjao Ye et Kristina E. Ward, "The Depiction of Illness and Related Matters in Two TopRanked Primetime Network Medical Dramas in The United States: a content analysis ", Journal of Health Communication, vol. 15, 2010, p. 555-570.

41. Amir Hetsroni, « If you Must be Hospitalized, Television is not the Place: Diagnoses, Survival Rates and Demographic Characteristics of Patients in TV Hospital Dramas ", Communication Research Reports, vol. 26, 2009, p. 311-322; Brian A. Primack, Traci Roberts, Michael Fine, 
Francesca Dillman Carpentier, Kristen Rice et Amber Barnato, «ER vs. ED: a Comparison of Televised and Real-Life Emergency Medicine», Journal of Emergency Medicine, vol. 43, 2012, p. 1160-1166.

42. Voir Beatrix Hoffman, "Emergency Rooms : the reluctant safety net », in History and Health Policy in the United States: Putting the Past Back in, ed. Rosemary Stevens, Charles E. Rosenberg, Lawton R. Burns, Rutgers University Press, 2006.

43. De manière surprenante, le docteur Shepherd semble confondre Medicare (le programme d'assurance hospitalière pour les personnes de plus de 65 ans) et Medicaid (le programme de soins médicaux pour les plus pauvres) dans cet extrait.

44. Dan Mangan, « Medical Bills Are the Biggest Cause of US Bankruptcies: Study », 25 juin 2013, CNBC [en ligne] https://www.cnbc.com/id/100840148 (consulté le 12 octobre 2018).

45. "This sucks! When I had insurance I could get hit by a bus or catch on fire you know! And it wouldn't matter. Now I gotta be careful ?» (traduction de l'auteure).

46. Aux États-Unis, on estime qu'environs 45000 personnes meurent chaque année, car leur police d'assurance maladie est trop peu généreuse. David Cecere, « New study finds 45,000 deaths annually linked to lack of health coverage », Harvard Gazette [en ligne], octobre 2009, https:// news.harvard.edu/gazette/story/2009/09/new-study-finds-45000-deaths-annually-linked-tolack-of-health-coverage/ (consulté le 12 octobre 2018).

47. Jean-Claude Soulages, Les rhétoriques télévisuelles : le formatage du regard, Bruxelles, De Boeck, 2007.

48. Sarah Kozloff, « Narrative Theory and Television », Channels of Discourse, reassembled : television and contemporary criticism, ed. Robert C. Allen, Chapel Hill, University of North Carolina Press, 2010, p. 67 [p. 67-100].

49. Ibid.

50. Christian Bosseno, «Les séries télévisées", Encyclopédie Universalis [en ligne], https:// www.universalis.fr/encyclopedie/series-televisees/ (consulté le 12 août 2018).

51. David Buxton, Les séries télévisées: forme, idéologie et mode de production, Paris, L'Harmattan, 2010.

52. Éric Maigret et Guillaume Soulez, "Les nouveaux territoires de la série télévisée ", Médiamorphoses, hors-série $n^{\circ} 3,2007$, p. 11 [ p. 7-13].

53. Joseph Belletante, Séries et politique. Quand la fiction contribue à l'opinion, Paris, L'Harmattan, 2011.

54. Jean-Pierre Esquenazi, Mythologie des séries télé, Paris le Cavalier bleu, 2009.

55. George Gerbner et Larry Gross, "Living with Television : the Violence Profile ", Journal of Communication, vol. 26, 1976, p. 172-194.

56. G. Gerbner, M. Morgan, N. Signorielli, « Programming, health portrayals, what viewers see, say and do ", in Television and Behavior, vol. 2 Technical Reviews, ed. D. Pearl, L. Bouthilet \& J. Lazar (éds.), Rockville, Maryland, 1982, p. 293 [p. 291-307] (traduction de l'auteure).

57. «Rating History: Grey's Anatomy », Fandom.com [en ligne], consulté le 23 décembre 2018 : « Does anyone see what happened. Once it got political, ratings dropped. " 


\section{RÉSUMÉS}

Cet article analyse "l'asymétrie réaliste » dans les séries télévisées médicales étatsuniennes, c'est-à-dire un choix de focalisation combinant l'hypervisibilité de l'expertise médicale et l'invisibilisation des inégalités d'accès aux soins médicaux. Plus précisément, l'article étudie l'essor du canon sériel médical et l'évolution historique des choix de focalisation de ce genre en s'appuyant sur un corpus de séries médicales produites entre 1950 et nos jours aux États-Unis. L'article étudie également les enjeux épistémiques de l'asymétrie réaliste dans ce genre très populaire et dans le contexte de réception étatsunien, où la question de l'accès aux soins médicaux demeure une inquiétude permanente, notamment pour les plus pauvres.

This article analyses the " asymetrical realism » of US medical TV series, understood as a choice of focalization combining the hypervisibility of medical expertise and the invisibilisation of inequalities in access to medical care. The article is based on a corpus of US medical series produced from the 1950s to this day. It tracks the rise and consolidation of the medical series canon as well as the historical evolution of focalization choices in the genre. The article analyses the epistemic stakes of asymetrical realism in this very popular genre, in the US reception context where the question of access to health care is a permanent source of concern, especially for the poorer segments of the population.

\section{INDEX}

Keywords : medical TV series, United States, health care system, medicine, doctors, focalisation, presentism, canon

Mots-clés : séries médicales, Etats-Unis, système de santé, médecine, médecins, focalisation, présentisme, canon

\section{AUTEUR}

\section{ELISABETH FAUQUERT}

Elisabeth Fauquert est normalienne de l'École Normale Supérieure de Cachan, agrégée d'anglais et docteure en civilisation des États-Unis. Elle est l'auteure d'une thèse sur la réforme de la protection maladie sous la présidence Obama, d'un manuel de civilisation des États-Unis (Éditions Armand Colin, 2019) et directrice de l'ouvrage collectif La présidence de Barack Obama (Éditions Atlande, Clefs Concours, 2019).

Elisabeth is a former student of the École Normale Supérieure de Cachan and agrégée d'anglais. She is the author of a PhD thesis which analyzed health care reform during the Obama presidency. She is the author of a textbook on American history and civilisation (Armand Colin, 2019), and the editor/co-author of La présidence de Barack Obama (Éditions Atlande, Clefs Concours, 2019). 